\title{
XXIII.
}

\section{Die subcutanen Injectionen des Alkohols und älnlich wirkender Stoffe bei Erkrankungen der Blntgefässe.}

\author{
Von Dr. Carl Schwalbe in Magdeburg.
}

Seit einer Reihe von Jahren habe ich in verschiedenen kleinen Aufsätzen und Vorträgen auf die schönen Heilerfolge aufmerksam gemacht, welche man durch subcutane und parenchymatöse Injectionen des Alkohols, der Jodtinctur, des Aethers und anderer ähnlich wirkender Stoffe erreichen kann. Die folgenden Mittheilungen bilden eine weitere Ausführung dieser Erfahrungen.

Bekanntlich machte zuerst B. v. Langenbeck (Berlin. klin. Wochenschrift, 1869, No. 12) darauf aufmerksam, dass man durch Einspritzungen von Ergotin, in Alkohol und Glycerin gelöst, in die Umgebung der Aneurysmen, die Aneurysmen verkleinern und heilen kann. Paul Vogt (Ueber die Behandlung der Varicen. Berl. klin. Wochensch. 1872. No. 10) hat die gleiche Behandlungsmethode auf Varicen angewandt und günstige Erfolge erzielt. Nach meinen Erfahrungen bei der Behandlung der Strumen (dieses Archiv 1871) konnte die Erklärung dieses Heilvorganges nicht zweifelhaft sein und ich äusserte mich in einem kleinen Aufsatze: „Ueber die parenchymatöse und subcutane Injection des Alkohols und ähnlich wirkender Stoffe" (dieses Archiv 1872) dahin, dass auch hier eine chronische Entzündung und Sclerose entstände und dadurch ỏie Heilung erzielt wird. Schon damals theilte ich einen Fall mit, bei welchem Varicen durch subcutane Injectionen von Alkohol in die Nähe des Varix geheilt wurden. 'Ich hatte Gelegenheit die Dauer der Heilung nach einem Jahre zu bestätigen. Diese Ansichten halte ich noch heute vollständig aufrecht und bringe in den folgenden Betrachtungen weitere Beweise dafiir.

Zunächst will ich auf die Einwürfe eintreten, welche gegen meine Auffassung der Ergotininjection'gemacht worden sind. v. Langenbeck bebt in einem längeren Vortrage, auf der Naturforscherversammlung in Wiesbaden gebalten, bervor, dass er $z u$ 
subcutanen und parenchymatösen Ergotininjectionen einige Male auch nur wässrige Ergotínlösung genommen und doch die gleiche Wirkung erzielt habe, wie mit der Lösung in Alkohol und Glycerin. Der Fall würde nur beweisend sein, wenn die Injection nicht in die Nähe des Aneurysmas, sondern an eine andere Stelle geschehen wäre. Auch die wässrige Ergotinlösung übt entzündlichen Reiz aus, öfter sogar ziemlich starken, wie die Erfahrungen vieler Aerzte lehren. So äussert sich Wernich, welcher eine grosse Érfahrung über Ergotininjectionen hat, dass in allen Fällen von subeutanen Injectionen die Injectionen heftigen Schmerz erzeugten, dass sich knötchenartige Verhärtungen bildeten, welche oft noch Monate lang persistirten und vielleicht mit Residuen der Einspritzung im Zusammenhang standen, welche nie vollständig resorbirt wird. (VirchowHirsch Jahresbericht 1873.)

Drasche beobachtete Röthung der Haut, bisweilen tagelang anhaltend, oft gelbgrünliche Entfärbung, öfter Schmerzen; später Induration, namentlich bei nicht filtrirten, wässrigen Lösungen, weniger bei Glycerinlösungen (Virchow-Hirsch Jahresbericht 1872). Buchheim (Virchow-Hirsch Jahresbericht 1874) zeigte, dass das Mutterkornextract eine stark saure Reaction hat, von Milchsäure herrührend ${ }^{1}$ ). Ich bestimmte in einem Extract, welches ich aus der Apotheke in Weinheim entnahm, die Milchsäure quantitativ und fand einen Gehalt von 0,09 freier Milchsäure auf 1,0 Grm. Extract. v. Langenbeck wandte bei dem Falle von Aneurysma, welches er mit wässriger Ergotinlösung behandelte, Extract. Secal. cornut. aquosi 2,5 in Aq. destill. 14,0 Grm. an (Gatiano, Ueber die subcutane Anwendung des Ergotins. Inaugural-Dissert. Berlin 1873). Dies würde einen Procentgehalt von 1,36 Milchsäure ergeben. Ich machte mit einer $\frac{1}{2}$ procentigen Milchsäurelösung in destillirtem Wasser subcutane Injectionen neben Varicen, erhielt eine deutliche Infiltration und Röthung der Haut, welche während mebrerer Tage bestand und bei einer Injection sogar zur Abscedirung führte. Der Beweis, dass das Extract. Secal. cornuti nicht örtlich reizend und chronische Entzutndung erregend wirkt, ist also noch nicht gebracht. Ebenso wenig ist aber durch die Dissertation von Catiano der Beweis ge-

1) Dragendorff hat in dem Mutterkornextract eine Süure gefunden, welche er Sclerotinsäure nennt. Die Constitution derselben scheint noch nicht fest zu stehen. 
liefert, dass Ergotin, an einer beliebigen und von erweiterten Gefässen weit entfernten Stelle eingespritzt, durch Vermittlung des vasomotorischen Centrums verengernd auf die Muscularis der erweiterten Gefässe wirkt. Catiano hat einen einzigen Fall citirt, wo man eine Wirkung auf von der Einstichsstelle weit entfernte Varicen durch Vermittelung des vasomotorisehen Centrums hätte erwarten sollen. Der Fall ist zu beweisend für mich als dass ich ihn übergehen könnte.

Eine Hochschwangere kommt mit Varicen beider unteren Extremităten in die Behandiung des Dr. Ruge. Nachdem zuerst die Varicen genau beschrieben werden, wobei hervorgehoben wird, dass dieselben links viel stärker waren, als rechts, fährt die Krankengeschichte fort, wie folgt: "Theils, weil Pat. durch eine in der ersten Schwangerschaft überstandene Blutung (in Folge eines geplataten Varix) die Gefahren einer solchen Blutung kennt, theils, weil ihr Hausarzt sie auf die Gefahren, die bei der Entbindung eintreten können, aufmerksam gemacht hat, theils, weil (allerdings namentlich zu Anfang der Schwangerschaft) entschiedene Beschwerden, Schmerzen, die Folge der Varicositäten waren, entschliesst sich Patientin zu einer Ergotinbehandlung. Die Lösung bestand aus:

Extract. secal. cornut. aquos. 2,5

Spiriti vini rectific.

Glycerini $\overline{\mathbf{a}} \mathbf{a} \mathbf{7}, \mathbf{5}$ davon je $\frac{1}{2}$ Spritze.

I. 12. April 1872: 0,06 Ext. sec. in die Mitte der Aussenfläche der beiden Oberschenkel subcutan - ambulant. 14. April: Pat. wurde bei ihrem Heimweg am 12. April von einem Fieberanfalle und so starken Schmerzen befallen, dass sie nach Hause fahren musste. - Sie wird in die Anstalt aufgenommen. - Im Ganzen schwach genährtes Individuum. Belde Oberschenkel etwas geschwollen, schmerzhaft. - Ruhige Lage im Bett.

II. 15. April: 0,06 Extr. sec. subcutan etwas oberbalb des Condylus ext. des linken Oberschenkels injicirt. 16. April: Die zweite Stichöffinung verursacht der Pat. noch ziemlich beträchtliche Schmerzen. Die ganze linke Extremitzt ist etwas empfiddich, so dass Pat. blos mit stelfer unterer Extremität gehen kann. Die Varicositäten haben ganz bedeutend abgenommen, namentlich die grosse Vene an der Aussenfläche des linken Oberschenkels; doch auch die Venen am Unterschenkel. Pat. ist entschieden mit ihrem Zustande trotz der Schmerzen sehr zufrieden.

III. 5 Uhr Nachmittags: 0,06 Extr. sec. subcutan in die Mitte des Vaterschenkels dicht nach aussen von der Tibia. 18. April: Die Umgebung der 2. und 1. Stichöffnung ist ziemlich stark geschwollen, geröthet, schmerzhaft. Der Erfolg ist sebr auffallend, jetzt ganz besonders an der Aussenseite des Unterschenkels bemerkbar. Dle zahlreichen hier befindlichen Varicosităten erscheinen kleiner, viel weniger oberflächlich, und anch in Zahl geringer. Doch ist auch der Erfolg an den grossen Venen des Unterschenkels sehr in die Augen springend. Pat. Iiegt allerdings ziemlich viel zu Bett, leidet indess grade an Durchfall, der sie oft 
nach dem Hofe heruntertrelbt. 19. April: Die circumscripte Anschwellung hat abgenommen.

IV. Morgen 12 Uhr: 0,06 Extr. sec. In die Aussenfläche des Unterschenkels (etwa Mitte).

B. Der Erfolg der Injection markirt sich noch dadurch in sehr eminenter Weise, als die Varicositäten an der nicht local behandelten rechten unteren Extremität (trotz des Liegens) gar nicht abgeschwollen sind.

25. April: Pat. hat sich während der letzten Zeit ziemlich viel in ihrem Bette befunden, weil namentlich die letzte Injectionsstelle und die vorletzte noch ziemlich geschwollen, lebhaft geröthet und bei Bewegungen sehr schmerzhaft sind. Zeitweise hat sogar 0edem des linken Unterschenkels bestanden. - Die Varicositäten an dem Iinken Unterschenkel haben ganz bedeutend abgenommen, die Varicositäten am Oberschenkel scheinen wieder etwas zuzunehmen; die Varicositäten an der rechten unteren Extremităt haben zwar im Ganzen auch etwas abgenommen, sind aber doch bedeutend stärker, als an der linken Extremität, obwohl sie früher von letzteren bedeutend übertroffen wurden. - Pat. ist mit dem Erfolge sehr zufrieden, wänscht bald auch am anderen Fuss behandelt zu werden.

v. $\frac{1}{2}$ Sprttze injicirt in den linken Oberschenkel an der Aussenfäche in der Mitte zwischen unterem und mittleren Drittel.

VI. 27. April Nachm. 5 Uhr: 0,06 Extr. sec. subcutan an die Innenfläche des linken Oberschenkels etwa in der Mitte. 29. April: Die beiden Punctionsstellen am Unterschenkel sind noch stark geröthet, geschwollen, schmerzhaft. Hier beiderseits deutliche Flactuation.

VII. 3. Mai: Injection von 0,05 Ext. sec. subcutan an die Aussenfläche des linken Oberschenkels (zwischen dem mittleren und unteren Drittel). 6. Mai: Die Varicen, die vor dem 3. Mal wieder stärker zu werden begannen, sind wieder enorm geschwunden, obwohl Pat. fast den ganzen Tag sich ausserhalb des Bettes befindet. Selbst die grösseren Venen an der Aussenfiäche des linken Oberschenkels sind wieder bedeutend kleiner geworden. Die 2 circumscripten gerötheten Stellen (fluctuirend) am linken Unterschenkel bestehen fort. Die letzte Injectionsstelle etwas infiltrirt. Die Venectasie an der rechten unteren Extremität bedeutend stärker als links; auf der rechten Sohle unangenehmes Jucken. An der linken unteren Extremität sonst weiter keine Beschwerden.

VIII. 8. Mai Morgens 0,1 Extr. sec. in den mittleren Thell der linken Wade. 10. Mai: Die Injectionsstelle in den Waden hat namentlich in den ersten Stunden so enorme Schmerzen veranlasst, dass Pat. das Weinen nicht unterdrücken konnte. Die Wirkung auf die Venen der linken nnteren Extremität ist nicht zu verkennen. Die Wade geröthet, geschwollen, schmerzhaft bei Druck. Die vorderste Injectionsstelle an der Aussenfläche des linken Oberschenkels zwischen dem mittleren und unteren Drittel ist noch geschwollen, geröthet, nicht mehr schmerzhaft. Die beiden Injectionsstellen an der vorderen Fläche des linken Unterschenkels markiren sich als kleine nicht fluctuirende Prominenzen. - Die Differenz zwischen den stärkeren Venectasien der rechten unteren Extremität und den jetzt schwächerender linken unteren Extremität ist aufallend.“ 
So weit Gatiano. Am 11. Mai 1872 trat die Geburt ein. Injectionen wurden nicht weiter gemacht.

Catiano macht nun folgenden Schluss: „Dieser Fall und derjenige von Aneurysma (bei welchem wässrige Ergotinlösung eingespritzt wurde, Schw.) beweist so prägnant die Wirkung des Ergotins, dass jeder Zweifel, ob dieses Mittel diese Wirkung bervorbringe, beseitigt ist. Schwalbe in Zürich meint, dass nicht das Ergotin, sondern der Alkohol und das Glycerin, welche meistentheils mit eingespritzt werden, als Erreger chronischer Entzündung und Narbenbildung die Heilung dieser Ectasien bewirke. Der Fall von Aneurysma wurde mit reinem Érgotin ohne Alkohol und Glycerin behandelt und, ohne dass eine schmerzhafte Stelle eintrat, verschwand das Aneurysma und in dem Falle von Varicen wurden die Injectionen nicht im Unterhautfettgewebe über die Knoten, sondern in entfernteren Punkten, wo keỉne Ectasien sich befanden, gemacht."6

Ich schliesse grade umgekehrt. Dieser Fall beweist, dass die Ergotinlösung als Ergotinlösung durch Wirkung auf die Muscularis der Venen nicht zur Heilung der Varicen beiträgt. Gatiano sagt Seite 12 seiner Dissertation: „Die Ansicht über die physiologische Wirkung dieses Mittels lässt sich dahin resumiren: dass es Contractionen der Gefässmuscularis bewirkt, welche Contractionen durch Vermittelung des vasomotorischen Centrums ausgelöst werden." Als These stellt derselbe ferner auf: "Die Contractionen der Gefässmuscularis bei der subcutanen Anwendung des Ergotins werden durch Vermittelung des vasomotorischen Centrums ausgelöst."

In der oben angefuhrten Krankengeschichte wurden die Injectionen an der linken unteren Extremität gemacht. Es wird in der Krankengeschichte gesagt: „Der Erfolg der Injection markirt sich noch dadurch in sehr eminenter Weise, als die Varicositäten an der nicht local behandelten rechten unteren Extremität (trotz des Liegens) gar nicht abgeschwollen sind! " ferner: „Die Venectasie an der rechten unteren Extremität bedeutend stärker als links." „Die Wirkung auf die Venen der linken unteren Extremität ist nicht zu verkennen." „Die Differenz zwischen den stärkeren Venectasien der rechten unteren Extremität und den jetzt schwächeren der linken unteren Extremität ist auffallend." Es wird also immer her 
vorgehoben, dass die Varicen der rechten Extremität durch die Einspritzungen an der linken nicht verändert wurden. Wo bleibt da die Wirkung des vasomotorischen Centrums? Andererseits wird bei den Einspritzungen immer bemerkt, dass sie Geschwulst, Entzündung erregten, welche tagelang bestand. An einer Stelle beisst es sogar: "Die Wade geröthet, geschwollen, schmerzhaft bei Druck." Und da soll keine örtliche Einwirkung der injicirten Flüssigkeit stattgefunden haben! Catiano sagt freilich, dass niemals über den Varix eingestochen wurde. Welcher vorsichtige Arzt wird das überhaupt thun! Man kann sich sehr leicht überzeugen, dass selbst bei schwachem Druck sich die Injectionsflüssigkeit auf grosse Entfernungen unter der Haut von der Injectionsstelle aus verbreitet. Besonders schön sieht man dies bei Geschwüren der Haut, wo man 2-3 Zoll rom Rande des Gesebwüres entfernt einspritzen und die Injectionsflüssigkeit aus dem Geschwüre aussickern sehen kann. Hueter (Die parenchymatöse Injection und Infusion der Carbolsäure. Deutsche Zeitschrift für Chirurgie IV. Bd. 5. u. 6. Heft) durchrieselt selbst bei schwachem Drucke grosse Strecken des subcutanen Bindegewebes mit Carbolsäure zur Heilung des Erysipelas und anderer Krankheiten. Aber die Wirkung des Ergotin auf die Muscularis der Venen ist noch nicht einmal sieher durch Experimente an Thieren festgestellt. In Virchow-Hirsch Jahresbericht 1874 Bd. I, S. 484 heisst es: "Nach Wernich wirkt das Mutterkorn nicht contrahirend auf die Muskelwand der Arterien oder iberhaupt auf organische Musculatur, wie man bisher im Allgemeinen annahm, sondern im Gegentheil herabsetzend auf den Tonus der Gefässe, vor Allem der Venen, so dass in den letzteren sich das Blut anhäuft, während die Arterien leerer werden und so einen secundären Contractionszustand zeigen. W. basirt seine Ansicht auf die mikroskopischen und makroskopischen Befunde an Versuchsthieren und auf eine kritische Betrachtung der mit Bezug auf den Blutdruck nach Ergotineinspritzungen angestellten Versuche, nach denen eine primäre Erhöhung des Druckes nicht bewiesen ist, vielmehr ein primäres Absinken des Druckes in den Arterien und dann erst eine allrnähliche Erhebung stattfindet, womit auch Wernich's eigenè manometrische Versuche übereinstimmen; endlich dass bei der Annahme einer activen Zusammenziehung der Arterien es unmöglich sein würde, die Heilwirkung von subcutanen Ergotininjectionen auf 
Aneurysmen physikalisch zu erklären. Den Effect der subcutanen Anwendung des Mittels bei Varicen und Mastdarmvorfall leitet Wernich her aus der vermehrten Thätigkeit des Herzens und secundären Beschleunigung in allen Gefässgebieten, welche dem primären Effecte zu folgen pflegt und stagnirenden Gefässinhalt in Bewegung $z u$ bringen geeignet ist."

Dieser Erklärung Wernich's über die Heilwirkung bei Varicen steht der Fall von Catiano erst recht entgegen, da man bei vermehrter Herzthätigkeit erst recht erwarten muss, dass beide Extremitäten von der Heilwirkung des Ergotin betroffen werden. Weiter auf die Erklärung der Ergotinwirkung einzugehen liegt nicht im Plane dieser Arbeit; jedenfalls sind noch keine übereinstimmende Resultate über die Wirkung des Ergotin auf die Gefässmusculatur erreicht. Soll Ergotin durch Vermittelung des vasomotorischen Centrums heilen, so muss es auch bei Anwendung durch den Magen wirken. Wenn bei dieser Anwendung Aneurysmen und Varicen heilen, dann bin ich widerlegt. Dass übrigens meine Ansicht nicht allein dasteht, zeigt ein Blick in Billroth's allgemeine chirurgische Pathologie und Therapie. Dort heisst es bei der Besprechung der Heilmethoden der Aneurysmen (7. Aufl. S. 656) „v. Langen beck injicirte in die unmittelbare Umgebung von Aneurysmen eine Lösung von Ergotin und erzielte auch dadurch Heilungen; ich erkläre mir die Wirkung dieser Behandlung auch dadurch, dass eine Entzündung der Gefässwand mit den oben erwähnten Folgen angeregt wird."

Schliesslich möchte ich noch einmal hervorheben, dass bei sehr vielen Aneurysmen grade die Tunica media, welche die Muskeln enthält, fehlt oder wenigstens sehr geschwunden ist (Förster, Pathol. Anatomie 2. Aufl. S. 717; Rindflejseh, Pathol, Gewebelehre 1. Aufl. S. 172; Quincke, Krankheiten der Arterien S. 415 in v. Ziemssen's Handbuch der spec. Patholog. u. Therap. I. Auff.). Nur der Fall von Wolff, Heilung eines Aneurysma aortae abdominalis durch Einspritzungen von Ergotin in die Regio mesogastrica würde für eine Ergotinwirkung auf die Muscularis des erkrankten Gefässes beweisend sein (Berliner klinische Wochenschrift 1873. No. 27).

Wenn man diese Krankengeschichte genau durchliest und ausserdem die Thatsachen erwägt, dass andere Autoren, Rouge, $Q$ u incke, keinen Erfolg von den Ergotineinspritzungen bei Aneurysmen der 


\section{8}

Bauchaorta erreichten, so wird man die Möglichkeit eines Irrthums in der Diagnose für wahrscheinlich halten.

Es liegt mir nun noch ob durch weitere Beispiele zu zeigen, dass Alkohol oder eine andere reizende Flüssigkeit, subcutan in die Nähe von erweiterten Gefässen eingespritzt, eine Entzündung erregt, deren Folge Verengerung und bei öfterer Wiederholung Schluss des Gefässes ist.

\section{Folgender Fall wird diese Wirkung am besten zeigen:}

1. Marie Schobess, 13 Jahre alt, aus Ried-Nordbausen bei Nordhausen. Vor einigen Jahren wurde eine cavernöse Geschwulst des Oberschenkels durch Professor Volkmann in Halle exstirpirt. Stat. praes.: Am rechten Arm und der rechten Schulter zeigten sich verschiedene venöse Angiome. A. Am vorderen Rande des Cucullaris ungefähr in der Höhe des Zungenbeins, $4 \mathrm{Cm}$. lang, $2 \mathrm{Cm}$. breit, blau durchscheinend, Inhalt lässt sich herausdrücken, Probepunction Blut. B. In der Fossa supraspinata dicht am Acromion eine pralle nicht blau durchscheinende Geschwolst, deren Inhalt sich ausdrücken lässt; Probepunction Blut; $3 \mathrm{Cm}$. lang, $2 \mathrm{Cm}$. breit. C. Dicht über der Pars acromialis claviculae blau durchscheinende, unregelmässig höckrige, weiche Geschwulst, comprimirbar, Probepunction Blut; Länge $6 \mathrm{Cm}$., Breite $3 \frac{1}{2} \mathrm{Cm}$. Die ganze Fossa supraclavicularis zeigte ein sehr stark ausgebildetes subcutanes Venennetz. D. Auf dem Ansatz des Musc. deltoideus eine pralle, blaue, comprimirbare, $4 \mathrm{Cm}$. lange, $3 \mathrm{Cm}$. breite Geschwulst. Punction Blut. E. In der Ellenbogenbeuge unregelmässig höckrige blaue Gescbwulst, $5 \mathrm{~cm}$. lang, $4 \mathrm{Cm}$. breit, bei Ruhe des Armes prall gefüllt, bei Bewegungen zum grössten Theil entleert, besonders wenn der Arm erboben wird. Punction Blut. F. Auf der Volarseite des Vorderarms an der Stelle, wo Vena mediana cephalica und Vena cephalica sich zu trennen pflegen, blaue, $2 \mathrm{Cm}$. lange und $1 \mathrm{Cm}$. breite Geschwulst. Punction Blut. G. Am Handgelenk neben der Art. ulnaris aus der Tiefe hervorkommende eine $2 \frac{1}{2} \mathrm{Cm}$. lange, $1 \frac{1}{2} \mathrm{Cm}$. breite nicht blau durchscheinende aber comprimirbare Geschwulst. Punction Blut. H. Unter der Fascla palmaris eine nur wenig hervorragende, $3 \mathrm{Cm}$. lange und $2 \mathrm{Cm}$. breite Geschwulst, welche sich leicht zusammendrücken lässt. An diesen cavernösen Gesclıwülsten wurden in die Umgebung folgende subcutane Injectionen gemacht.

A. 8. Juli 1 Spritze Alkohol von 60 pCt.

$\begin{array}{llllc}\text { 11. - } & - & - & - & - \\ \text { 16. - } & - & - & - & 50 \mathrm{pCt} . \\ \text { 21. - } & - & - & - & - \\ \text { 28. - } & - & - & - & - \\ \text { 4. Aug. } & - & - & - & - \\ \text { 14. - } & - & - & - & - \\ \text { 21. - } & - & - & - & - \\ \text { 31. - } & - & - & - & -\end{array}$

5. Sept. geheilt, man sieht nichts mehr von einer Geschwulst und fühlt nur noch eine kleine harte Stelle. 


\section{9}

B. 8. Jull 1 Spritze Alkohol von $60 \mathrm{pCt}$.

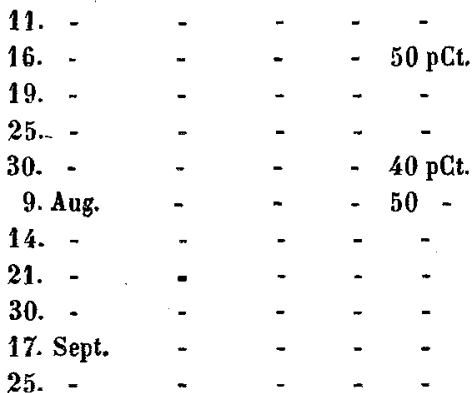

Anfang October ist noch eine etwa bohnengrosse fluctuirende Geschwulst ganz in der Tiefe zu fühlen. Die Injectionen machten an dieser Stelle immer so starke Schmerzen, dass von vollständiger Heilung abgestanden warde.

C. 7. Juli 1 Spritze Alkohol von $60 \mathrm{pCt}$.

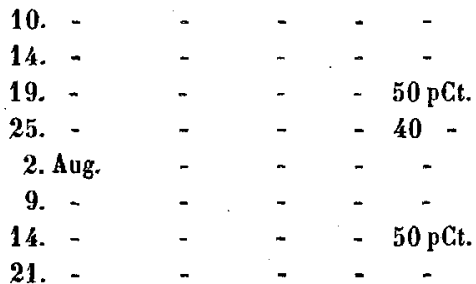

Anfang September Geschwulst verschwunden; man fühlt noch einige kleine Buckel ähnlich wie weiches Fettgewebe.

D. 7. Juli 1 Spritze Alkohol von 60 pCt.
10. -
14. -
23.
28. -
30. - - - $\quad-40$ pCt.
6. Aug. - - - $\quad-50$ -
11.
18.
25. -
28. -
30.
9. Sept.
10.
12. -
15. -
18.
19.
- 70 pCt. 
26. Sept. 1 Spritze Alkohol von 50 pCt.

4. 0ct.

- 70 -

8. 0ct. vollständig geheilt, nur noch eine leichte Härte zu füblen.

E. 3. Juli 1 Spritze Phenylalkohol 2 pCt.

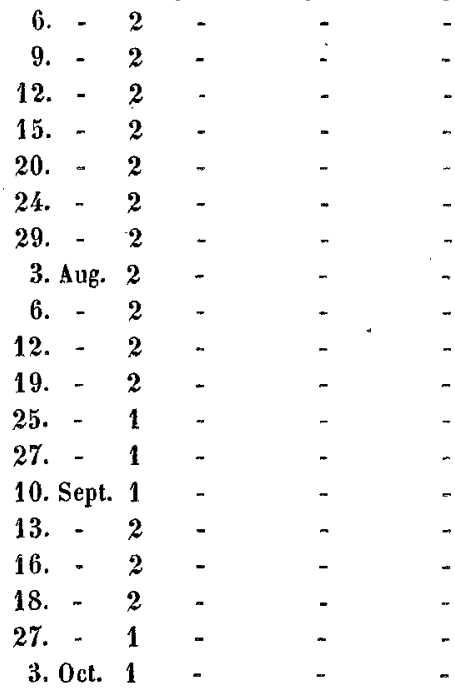

8. 0ct. vollständig geheilt. Haut über der Geschwalststelle vollständig normal; man füblt noch einige unbedeutende Verhärtungen.

F. 3. Jull 1 Spritze Alkohol 60 pCt.

7. -
$10 .-$
$23 .-$
$13 . \quad-$
$23 .-$

Ende August vollständig verschwunden, man fühlt der Vena mediana cephalica entsprechend einen dünnen barten Strang unter der normalen Haut.

G. 4. Juli 1 Spritze Alkohol $50 \mathrm{pCt}$.

$\begin{array}{llll}8 .- & - & - & 60- \\ 11 .- & - & - & 60- \\ \text { 18. - } & - & - & 50- \\ \text { 25. - } & - & - & 40- \\ \text { 4. Aug. } & - & - & 50- \\ \text { 13. - } & - & - & 60- \\ \text { 30. Sept. } & - & - & 50-\end{array}$

Die Einspritzungen wurden mit grosser Vorsicht unmittelbar über der Geschwulst gemacht, um Arteria und Nerv. ulnaris möglichst zu schonen. Anfang October bestand noch eine bobnengrosse Geschwulst, deren Inbalt sich nicht ausdrücken liess. 
Ich stand vou weiterer Verkleinerung $a b$, weil bei jeder Injection der Nerv. ulnaris mehr oder weniger getroffen wurde, für einige Tage stark schmerzte und in seinem Bereiche leichte Anästhesie zeigte. Das Angiom H wurde nicht behandelt, weil es keine Beschwerden machte und weil ich die vielen Sehnen fürchtete, welche nothwendiger Weise getroffen wären.

Die Herren Dr. Hasse in Nordhausen und Dr. Hadlich in Heidelberg haben diesen Fall vor und nach der Behandlung gesehen.

Betrachten wir nun die Erscheinungen, welche man bei subcutanen Alkoholeinspritzungen beobachten kann, so ist Folgendes zu bemerken: Der Einstich wird im Anfang ungefähr $3 \mathrm{Cm}$. von dem Gefäss entfernt in der Richtung gemacht, dass die zu injicirende Flüssigkeit nach dem Gefäss hinströmt. Die Canüle wird zuerst allein eingestossen, um sich zu überzeugen, dass kein. Blutgefäss getroffen wird und dann die Spritze vorsichtig eingesetzt oder eingeschroben und darauf geachtet, dass sich die Canüle nicht verschiebt. Darauf wird je nach Umständen eine halbe oder ganze Spritze langsam eingespritzt. Spannt sich die Haut stark über der injicirten Flüssigkeit, so höre man sogleich mit der Injection auf, besonders wenn die betreffende Hautstelle ganz weiss werden sollte. Nun wird die Canüle schnell ausgezogen, die Oeffnung mit dem Finger so lange zugehalten, als noch Injectionsflüssigkeit oder einige Tropfen Blut ausfliessen. Den Einstich durch ein Pflaster zu schliessen, ist überflüssig. Der Schmerz nach der Alkoholinjection dauert in verschiedener Intensität gewöhnlich 1-2 Minuten. Concentrationsgrad des Alkohols, Geschwindigkeit der Injection, Nervenreichthum der betreffenden Gegend $u$. s. w. stufen denselben sehr verschieden $a b$. Nach der Einspritzung röthet sich die betroffene Hautstelle, schwillt an und fängt häufig von Neuem an etwas zu schmerzen, jedoch unbedeutend. Die Anschwellaing kann sich, besonders im Gesicht, recht weit über das Injectionsgebiet hinaus erstrecken. Nach wenigen Tagen sind aber alle Erscheinungen verschwunden mit Ausnahme einer leichten Verhärtung des subcutanen Gewebes. Noch 5-8 Tage nach der Injection kann man an der gleichen Stelle wieder eine Injection machen. Bei dem oben angeführten Fall habe ich die Geschwülste nach und nach mit einem Kranz von Injectionen umgeben und bin mit der Einstichsstelle 
immer näher gerückt. Treten Schmerzen und starke Schwellung nach der Einspritzung auf, so werden kalte Aufschläge gemacht; bei sehr starker Reaction nimmt man den Eisbeutel. Diese therapeutischen Maassregeln sind jedoch nur in seltenen Fällen nöthig. Sehr schön liess sich in dem oben angeführten Fall die allmähliche Verengerung des Lumens der Angiome und endliche Verödung nachweisen, ohne dass es zu einer Gerinnung des Blutes in demselben kam. Die fluctuirende, wegzudrückende Stelle wurde immer kleiner, bis sie zuletzt ganz verschwunden war. Damit war aber auch fast jede Geschwulst verschwunden. Alte, mit geronnenem Blut gefüllte Varicen sieht man sehr deutlich durch die Haut blau durchschimmern. Hier war keine Spur von blauer Färbung zu sehen. Nur bei der Geschwulst $G$ ist wahrscheinlich eine Gerinnung eingetreten, da sich der Inhalt zuletzt nicht mehr ausdrücken liess. Die tiefe Lage des kleinen Angioms machte eine genaue Beobachtung unmöglich.

Jedenfalls ist es die Regel, dass keine Coagulation in den zu heilenden, erweiterten Gefässen stattfindet. Die Verfärbung, durch die Injectionen erzeugt, wird nach und nach wieder resorbirt und in den meisten Fällen bemerkt man nach 1-2 Monaten keine Spur mehr von der Injection ausser der betreffenden Heilwirkung. Die Resorption des provisorischen Callus bei einer gut geheilten Fractur zeigt in wie grossartiger Weise der Organismus in Stande ist, die Producte chronischer Entzündung wieder zu entfernen.

Von unangenehmen Erscheinungen nach der Injection habe ich nur sehr wenig beobachtet. Bei über 3000 subcutanen Injectionen mit Aethylalkohol in verschiedenen Concentrationsgraden (15 bis $80 \mathrm{pCt}$.) habe ich nur 8 mal Eiterung beobachtet. 2 mal an einem Tage kam Eiterung nach Einspritzungen vor, welche wahrscheinlich mit einer nicht ganz sorgfälltig gereinigten Spritze gemacht waren. Meine Sprechstunde war sehr überfüllt und so wurde in der Eile die nothwendige sorgfältige Reinigung unterlassen. Beide Eiterungen, die eine im Gesicht, die andere unter der Haut des Knicgelenks, verliefen günstig und hatten einen sehr guten Heilerfolg. In zwei anderen Fällen hatte ich die Canüle mit angeschraubter Spritze eingestossen, ein subcutanes Blutgefäss angestochen und einen Bluterguss unter die Haut erhalten, welcher vereiterte. Bei der Oeffrung des Abscesses lag in der Mitte desselben der geron- 
nene Blutklumpen. Drei Fälle von subcutaner Eiterung erzeugte ich bei Inguinalhernien durch schnelle Aufeinanderfolge der Injectionen. Der Druck des Bruchbandes scheint den Eintritt der Eiterung zu begünstigen. Der achte Fall von Eiterung betraf ein chronisches Unterschenkelgeschwür. Hier trat die Eiterung wahrscheinlich auch dadurch ein, dass die Einspritzungen in das entzündete Gewebe zu schnell hintereinander gemacht wurden. Die Einspritzung, nach welcher Eiterung entstand, wurde unter das Geschwür gemacht; es bildete sich ein tiefliegender subcutaner Eiterheerd, welcher mit seiner Ausheilung auch das Geschwür zur vollständigen Heilung brachte. Die Erscheinung, dass bisweilen bei zu starker Ansammlung der Injectionsflüssigkeit ganz dicht unter der Haut die Haut an dieser Stelle ganz weiss wird, habe ich schon erwähnt. Verschwindet diese weisse Farbe nicht bald, indem man vorsichtig die Flüssigkeit durch Reiben unter die Haut zu vertheilen sucht, so wird ein Stückchen der Haut entsprechend der bleibend weiss gefärbten Stelle necrotisch und stösst sich ab. Es ist mir dieses ganz unschädliche Ereigniss 5 mal vorgekommen. In einem von diesen fünf Fällen wurde ungefähr ein thalergrosses Stück neerotisch. Der Fall betraf eine alte Frau mit sehr zahlreichen Varicen am Unterschenkel und sehr welker pergamentartiger Hąut. Aber auch hier heilte das Geschwür gut und hatte günstigen Einfluss auf die nahe liegenden Varicen. Die Zahl von 3000 subcutanen Injectionen mag Vielen sehr gross scheinen. Dieselbe ist leicht verständlich, wenn ich bemerke, dass ich eine Zeit lang bei allen Rheumatismen und Neuralgien, wo man Morphiuminjectionen macht, Alkoholinjectionen vornahm und noch jetzt in allen Fällen wo Jodtinctur äusserlich angewandt wird Alkohol subcutan gebrauche. Es stellen sich also meine Resultate mit Aethylalkoholinjectionen in Bezug auf Eiterung bedeutend günstiger als diejenigen Hueter's mit Phenylalkohol. Hueter hatte auf 1000 Injectionen 10 Eiterungen. Eine weitere, unangenehme Erscheinung, welche im Ganzen aber nur selten eintritt, ist eine vorübergehende Anästhesie der von der Injectionsflïssigkeit betroffenen Nerven. Ich habe in einem Falle eine Anästhesie des Nerv. digital. dorsal. des Daumens beobachtet, welche 14 Tage anhielt. Hier hatte die Injection direct den Nervenstamm getroffen. Ueble Folgen blieben nicht zurück. Des Falles ron Anästhesie des Ulnaris (Schobess) habe ich schon gedacht. Weiter 
ist mir von irgend welchen Nebenwirkungen nichts vorgekommen ${ }^{1}$ ). $\mathrm{Zu}$ den subcutanen Injectionen wurden, wie schon erwähnt, Flüssigkeiten von dem versehiedensten Alkoholgehalt benutzt. Die schwächste Goncentration war 15 Gewichtstheile Alcohol. abs. auf 85 Aq. destill. Die stärkste Concentration Alc. absol. Letzterer wurde aber nur sehr selten angewendel, da er am stärksten reizt und am meisten schmerzt. Am häufigsten bediente ich mich der Concentrationen von $40-60-80$ Gewichtstheilen Alc. abs. auf 60-40-20 Aq. destill. Je mehr Alkohol desto stärker der Schmerz, desto stärker die Reaction, alle übrigen Bedingungen natürlich als möglichst gleich gesetzt. Nach diesen allgemeinen Betrachtungen über die Wirkung der subcutanen Alkoholinjection kehre ich zu den speciellen Fällen del Gefässkrankheiten zurück. Schon oben erwähnte ich eines geheilten Falles von Varicen am Malleolus intern. Einzelne Varixknoten wurden von mir gewöhnlich durch 3-10 Einspritzungen geheilt.

Ausserdem habe ich noch mehrere Fälle von Varicen mit gutem Erfolg behandelt, in so fern als die Varicen kleiner wurden und die secundären Symptome, Schmerzen, Schwellungen u. s. w., aufhörten. Bis zur vollständigen Obliteration der Venen habe ich es in diesen Fällen absichtlich nicht getrieben. Ueberhaupt ist es in manchen Fällen vortheilhafter, die Contraction der Varicen nicht bis zur Obliteration fortzusetzen. Dahin gehören die Fälle, wo eine sehr grosse Anzahl von Venen und über grosse Strecken varicös erweitert sind und ferner jene Fälle, wo viele Venen verkalkt und thrombosirt sind, wo in Folge dieser bedeutenden Circulationsstörungen die Haut sehr atrophisch und welk ist. Hier würde die Obliteration noch

1) Dìese anăsthesìrende Wirkung der Alkohol-Injeetionen anf einen Nervenstamm wurde von Dr. Hasse in Nordbausen bei Bebandlung eines Neuroms des Nerv. medianus in der Mitte des Oberarmes verwerthet. Etwa 5 Injectionen von je 1 bis 2 Spritzen Spiritus in die Umgebung des spindelförmigen circa $5 \mathrm{Cm}$. langen und $1 \mathrm{~cm}$. im Durchmesser baltenden ungemein schmerzhaften Neuroms, mit dem taubes Gefübl in der Hand und sehr wesentliche Functionsstörungen des ganzen Armes verbunden waren, setzten die Schmerzhaftigkeit bedeutend herab, verbesserten die Gebrauchsfähigkeit des Armes nnd hatten eine merkliche Verkleinerung der Nenbildung zur Folge.

Die bereits im vorgerückten Alter stehende und ziemlich schwächliche Patientin war mit dem erreichten Resultat soweit zufrieden, dass von einer Fortsetzung der Kur Abstand genommen wurde. 
wegsamer Venen nur noch neue Circulationshindernisse setzen und die Errährung der Haut nur noch mehr erschweren. Die grösste Zahl dieser Fälle findet sich natürlich unter den alten Leuten; ich babe aber selbst bei einem Manne in dem kräftigsten Alter, 40 Jahre, und von kräftiger Constitution durch subcutane Einspritzungen oberhalb eines Unterschenkelgeschwürs, dessen Umgebung so stark infiltrirt war, dass man die Venen weder sehen noch füblen konnte, Verschlimmerung des Ulcus chronicum erreicht. Electrische Geisselung und Ruhe, so wie energische Aetzung des torpiden Geschwürs mit der Kathode eines constanten Stromes erwirkten Heilung. Als die Infiltration verschwunden war, fühlte man viele verhärtete und verkalkte Venen. In solchen Fällen ist die zuerst von mir empfohlene electrische Geisselung anzuwenden (Electrotherapeutische Beiträge, dieses Archiv Bd. 63, 1875). Reicht dieselbe nicht aus, so wählt man einige der unbequemsten und schmerzhaftesten Varicen und verkleinert dieselben so lange durch subcutane Einspritzungen bis die lästigen Symptome verschwinden. Bei allen Arten von Varicen trägt die Geisselung wesentlich zur Besserung bei. Elastische Strümpfe lasse ich nur im äussersten Nothfall tragen; ich habe dieselben bis jetzt nur in einem Falle angewandt und in diesem entstand trotz des regelmässigen Tragens der gut gearbeiteten Strümpfe ein Ulcus chronicum. Varicen während der Schwangerschaft mit Einspritzungen zu behandeln balte ich für gewagt und unzweckmässig, weil man nie genau bestimmen kann, welche Varicen auch nach der Geburt fortbestehen und Beschwerden machen werden. Auch die electrische Geisselung wird besser unterlassen, weil sie wehenerregend wirkt.

.In letzterer Zeit hatte jch Gelegenheit eine Varicocele mit subculanen Alkoholinjectionen in die Ungebung der erweiterten Venen au behandeln und erbielt einen sehr schönen Heilerfolg. Nach 6 Injectionen eines 40 procentigen Alkohols in Zeiträumen von ungefähr 8 Tagen waren alle Beschwerden verschwunden und die Varicocele sehr bedeutend kleiner geworden. Ein Suspensorium war nicht mehr nöthig.

Aucb Dr. Hasse in Nordhausen hat bei der Behanalung der Varicen nach meiner Methode sehr gute Erfolge erzielt. Die kurzen Mittheilungen, welche er mir machte, sind folgende: 
C. Höche, 45 Jabre alt, Schuhmacher in Nordhausen. Varices dolorosae der Wade. Die Varices waren nicht sehr stark ausgebildet nur etwa von Rabenfederkielstärke, aber so schmerzhaft, dass Pat. kaum noch sitzen konnte und ihm seine Arbeit sebr erschwert wurde. Es wurde zinächst an der schmerzhaften Stelle die Injection gemacht - 2 Spritzen -; die Schmerzen traten darauf an den Injectionsstellen fast gänzlich zurück und wurden so nach und nach die übrigen demnächst am heftigsten schmerzenden Stellen bedacht. Jetzt am 23. Juni ist das blaue Venennetz ron der Oberfläche der Wade ganz verschwunden, doch fühlt man im Pan. niculus noch Phlebolithen. Dagegen zeigt sich jetzt Schmerz oberhalb der Poplitea (an der hinteren Fläche des Oberschenkels). Es wird daselbst eine Spritze injisirt. - Genesung.

Injectionen wurden gemacht mit 1 Theil Aether auf 100 Theile Brennspiritus am 28. April, 5., 12., 18. Mal, 23. Junl 1874.

Fran Cantorin Dreiling aus Herreden. Varices dolorosae pedis. 5. Juni 1874 Injection. Erhebliche Besserung.

Ferner schreibt mir Hasse unter dem 18. October 1875: „Varicen habe ich in der letzten Zeit in einer ganzen Reihe von Fällen mit sehr gutem und in einigen Fällen vollständigem Erfolge mit den Injectionen behandelt! "*

Ueber Teleangiectasien habe ich keine eigenen Erfahrungen. Hasse hat mir folgende Mittheilungen gemacht:

„Kleine Injectionen in die Umgebung derartiger Neubildungen bringen das Wachsthum zum Stehen, haben aber keinen wesentlichen Einfluss auf die Verkleinerung. Sie sind also in vielen Fällen sehr angebracht, wenn man Veranlassung hat eine eingreifende Operation (Excision) noch hinauszuschieben.

Injectionen in das Bereich der Teleangiectasie selbst verursachen in der Regel, namentlich wenn unter starkem Drucke eine verhältnissmässig reichliche Menge Spiritus injicirt wird, zunäcbst vollständiges Weisswerden der Neubildung, bedingt durch Auspressen des Blutes aus den Gefässen. Dadurch wird mitunter circumscripte Necrose bervorgerufen, ein Vorgang wie solcher oben schon besprochen worden ist. Mit der Vernarbung des Defectes pflegt dann auch die Teleangiectasie zu verschwinden.

In weniger brusque behandelten Fällen hat jedoch auch die bis zum partiellen Weisswerden der Geschwulst getriebene Injection keine Necrose, sondern nur allmählich fortschreitende subcutane Narbenbildung zur Folge, welche zwar Anfangs mit Einziehung der entsprechenden Stellen nicht allein unter das Niveau der noch stehenden Geschwulstreste, sondern auch unter das der benachbarten ge- 
sunden Haut einhergeht; nachdem jedoch die letzten Reste der Teleangiectasie verschwunden, nimmt die Haut nach und nach auch hier wieder ibre vollkommen normale Beschaffenheit an. Am zweckmässigsten scheint es zu sein, wo die Oertlichkeit solchẹs gestattet: z. B. an der Wange, die Canüle in dem Panniculus bis unter die Neubildung vorzuschieben, so dass die Injectionsflïssigkeit von unten her die Geschwulst umspülen und durchdringen kann.

Einige Krankengeschichten mögen zur Erläuterung des Gesagten dienen.

1. Runde Teleangiectasie der Kopfschwarte auf dem Scheitel, $1 \frac{1}{2} \mathrm{Cm}$. im Durchmesser, bel der 6 Monate alten Tochter des Posamentier Ruprecht in Nordhausen. Die ersten beiden Injectionen von officinellem Spiritus mit Zusatz von 1 pCt. Aether wurden nur in die Umgebung der Geschwulst gemacht - am 13. und 21. Januar 1874. - Es trat unmittelbar danach starke Anschwellung der Venen in der Umgebung ein. Die Geschwulst verkleinerte sich langsam. Bei der 3. und 4. Injection - am 24. Januar und 10. Februar - wurden auch in das Gebiet der Teleangiectasie selber einige Tropfen injicirt. Danach machte die Rïckbildung der Geschwulst schnelle Fortschritte und zwar inselartig von den Injectionsstellen ausgehend, $d . h$. es entstanden an diesen Punkten weisse etwas narbig eingezogene Inseln, welche an Umfang wuchsen, schliesslich in einander flossen. Seit der 5. Injection - am 2. März 1874 ist keine weitere gemacht worden, da die Rückbildung stetig fortschritt, so dass am 25. Juni nur noch einige nadelknopfgrosse hellrothe Pünktchen die Stelle bezeichneten, wo die Teleangiectasie sich früher befand. Einige Monate später waren aucb diese verschwunden, und das Endresultat 1st schliesslich ein sehr gutes, indem jetzt auch nicht einmal die Stelle mehr aufufinden ist, wo die Teleangiectasie ihren Sitz hatte.

Im Verlaufe der letzteren Jahre wurde eine ganze Reihe weiterer Fälle von Teleangiectasien mit demselben zufriedenstellenden Erfolge von mir behandelt; darunter auch solche, wo nach nicht vollständig gelungener Excision Reste der Geschwulst zurückgeblieben waren und sich wieder vergrössert batten.

Weniger gut schien das Verfahren anfangs in folgendem Falle sich zu bewähren.

2. Etwa thalergrosse Teleangiectasie auf der Bauchhaut des 1 Jahr alten Kindes des Ingenieur $\mathbf{D}$. in Nordhausen. Es wurden hier im Ganzen 8 Injectionen in die Umgebung und unter die Neubildung gemacht and zwar 1 im Juli, 4 im August, 2 im September und 1 im Norember 1875. Die Rückbildung erfolgte dabei so langsam, dass von dem Verfahren Abstand genommen wurde. Es war bis dahin nur ein stellenweises leichtes Abblassen der Geschwnlst, sowie sehr geringe Abnahme an der Peripherie wahrzunebmen. 
Als ich mich einige Jahre später nach dem Kinde erkundigte, wurde mir von der Mutter desselben mitgetheilt, dass die Neubildung seit jener Zeit stetig mehr und mehr abgeblasst und jetzt so gut wie verschwunden sei. Bei der daraufhin von mir selbst vorgenommenen Untersuchung fand ich die Stelle der früher selur grossen Teleangiectasie nur noch durch einige schmale blasșrothe Streifen angedeutet.

Auch ein Fall aus neuester Zeit, welcher gegenwärtig noch in Behandlung ist, mag hier noch Platz finden.

3. Fünfpennigstückgrosse Teleangiectasie auf der linken Wange der $\frac{\pi}{2}$ Jałr alten Tochter des Maurer Rüdiger zu Leimbach bei Nordhausen.

Am 17. November 1878: Erste Injection von $\frac{1}{3}$ Spritze des gewöhnlichen offcinellen Spiritus mit Zusatz von $1 \mathrm{pCt}$. Aether in das Fettpolster der Wange unter der Geschwalst. Letztere wird weiss verfärbt.

24. November: 2. Injection in derselben Weise.

6. Januar 1879: Es haben sich im Bereiche der Neubildung elnige kleine subcutane Narbeninseln mit Elnziebung der Oberläche gebildet. 3. Injection.

5. März: Eine breite Zone der Geschwulst ist beseitigt und die Haut hier narbig eingezogen.

Schliesslich sei eines relativ übel verlaufenen Falles gedacht.

Koch, Kind, 6 Monate alt, Nordhausen. Die Teleangiectasie, welche sich schon einer cavernösen Venengeschwulst näherte, nahm fast den ganzen Saum der Unterlippe ein, so dass an beiden Seiten kaum ein Strohhalm breit bis zu den Mundwinkeln frei war, sie erstreckte sich aber auch nach unten über die Cutis bis ziemlich zur Mitte zwischen Lippensaum und Kinn. Es wurde jederseits ungefähr $\frac{1}{2}$ Spritze injicirl, links etwas mehr als rechts. Nächste Folge war, dass hier die Lippe anfangs sehr blass wurde, dabei stark geschwollen war, und ein Stück des Llppensaumes (Schleimhautzellgewebe und Muskel) gangränös wurde; doch heilte der Defect mit guter, nicht entstellender Narbe. Die Teleangiectasie hat sich seit dieser ersten und einzigen Injection stetig verkleinert, besonders in ihrer linken Hälfte, so dass die Eltern bis jetzt ihre Zustimmung zu einer 2. Injection verwelgert haben, da sie solche vicht nöthig fanden!"

Ich habe durch Galvanolyse in zwei Fällen von Teleangiectasie am unteren Augenlide sehr schöne Erfolge erzielt, Heilung obne Narbencontraction, ohne nachtheiligęn Einfluss auf die Stellung des unteren Lides, obgleich das ganze untere Lid von der Erkrankung betroffen war. Die Behandlung dauert aber sehr lange.

Auch Althauss in London empfiehlt die Galvanolyse bei Teleangiectasien dringend.

Herr Dr. Wolf in Mannheim theilte mir brieflich 2 Heilungen von Varicen durch Alkoholinjectionen mit.

Schliesslich verdiente noch die Anwendung der subcutanen und submucösen Injectionen von Aethylalkohol und Phenylalkobol bei 
Hämorrhoiden einer besonderen Erwähnung. Auch hier leisten die Injectionen sehr gute Dienste. Dieselben werden an der Uebergangsstelle der äusseren Haut in die Mastdarmschleimhaut gemacht. Beim Einstechen der Canüle müssen natürlich alle Vorsichtsmaassregeln angewandt werden, um nicht in eine Vene zu spritzen. Bei der grossen-Empfindlichkeit dieser Gegend gegen Schmerz ist es am besten im Anfang Phenylalkohol in 2-3procentigen Lösungen zu nehmen. Bisweilen reicht eine einzige Einspritzung aus, um die Tästigen Symptome der Hämorrhoiden zu beseitigen. Zur Heilung sind natürlich oft zahlreiche Injectionen nöthig. Anästhesiren des Patienten dürfte häufig zu empfehlen sein.

\section{XXIV. \\ Kleinere Mittheilungen.}

1.

Zur Physiologie der Schweisssectetion.

Von Dr. B. Luchsinger,

Prof. der Physiologie an der Thierarzneischnle in Bern.

Herr Dr. A. Adamkiewicz hat sich veranlasst gesehen, auf einige, noch dazu möglichst schonende, hritische Bemerkungen von mir ${ }^{1}$ ) über selne Monographie "Die Secretion des Schweisses, eine bilateral-symmetrische Nervenfunction" im Tone gekränkter Unschuld zu erwidern ${ }^{2}$ ). Die folgenden Zeilen mögen darthnn, wie viel Gewinn er sich damit hat holen können.

I. Herr Adamkiewicz klagt mich an, seine Thatsachen über den directen cerebro-spinalen Ursprung eines Theiles der Schweissnerven „wider jeden literarischen Brauch" ignorirt, dieselben darauf ,grossmüthig" Vulpian zugestanden zu haben.

Nun, was sind denn die Thatsachen des Herro Adamkiewicz?

Man halbirt ein eben getödtetes Kätzchen zwischen Brust- und Lendenmark und entfernt sämmtliche Eingeweide, bis ein Präparat entsteht, das nur aus der Lendenwirbelsăule mit ihrem Mark, aus den Hinterpfolen und den zu ihnen tretenden Nervenplexus besteht.

I) Arch. f. d. ges. Physiol. Bd. XVIII, 1878. 488.

2) Arch. f. pathol. Anat. u. Physiol. Bd. LXXV. 1879. $555-560$. 